\title{
Beckwith-Wiedemann syndrome caused by maternally inherited mutation of an OCT-binding motif in the IGF2/H19-imprinting control region, ICR1
}

\author{
Rebecca L Poole ${ }^{1,2}$, Donald J Leith ${ }^{1,2}$, Louise E Docherty ${ }^{1,2}$, Mansur E Shmela ${ }^{3}$, Christine Gicquel ${ }^{3}$, \\ Miranda Splitt ${ }^{4}$ I Karen Temple ${ }^{1,2}$ and Deborah JG Mackay ${ }^{\star, 1,2}$
}

The imprinted expression of the IGF2 and $\mathrm{H} 19$ genes is controlled by the imprinting control region 1 (ICR 1 ) located at chromosome 11p15.5. DNA methylation defects involving ICR1 result in two growth disorders with opposite phenotypes: an overgrowth disorder, the Beckwith-Wiedemann syndrome (maternal ICR1 hypermethylation in 10\% of BWS cases) and a growth retardation disorder, the Silver-Russell syndrome (paternal ICR1 loss of methylation in $60 \%$ of SRS cases). In familial BWS, hypermethylation of ICR1 has been found in association with microdeletion of repetitive DNA motifs within ICR1 that bind the zinc finger protein CTCF; but more recently, ICR1 point mutations were described in BWS pedigrees. We present a case report of two brothers with BWS and prolonged post-pubertal growth resulting in very large stature. A maternally inherited point mutation was identified in ICR1 in both brothers, which altered binding of OCT transcription factors. The same mutation was present on the paternally inherited allele of their unaffected mother. This is a second report of a point mutation causing ICR1 hypermethylation by altering an OCT-binding motif. The atypical growth phenotype of the brothers may be connected to the unusual underlying cause of their BWS.

European Journal of Human Genetics (2012) 20, 240-243; doi:10.1038/ejhg.2011.166; published online 24 August 2011

Keywords: Beckwith-Wiedemann syndrome; IGF2/H19 ICR1; OCT-binding site; DNA methylation; imprinting disorder

\section{INTRODUCTION}

Human chromosome 11 p15 contains two clusters of imprinted genes that are critical regulators of early human growth, each expressed under the control of an imprinting control region (ICR). Genetic and epigenetic mutations of these genes cause Beckwith-Wiedemann syndrome (BWS: OMIM 130650), an overgrowth disorder, or Silver-Russell syndrome (SRS: OMIM 180860), which is characterised by growth restriction.

Clinical features of BWS include prenatal overgrowth, macroglossia, abdominal wall abnormalities, ear pits and creases, organomegaly, neonatal hypoglycaemia and elevated tumour risk. ${ }^{1}$ Molecular abnormalities associated with BWS include: paternal uniparental disomy (20-25\%), numerical and chromosomal abnormalities of chr11p15 ( $<1 \%)$, hypomethylation of ICR2 (60\% of cases), maternally inherited mutation of CDKN1C (5\%) and hypermethylation of ICR 1 ( $10 \%$ of cases). ${ }^{1}$

ICR1 directs parent of origin-specific expression of insulin-like growth factor 2 (IGF2). IGF2 is normally expressed only from the paternal allele, while the untranslated $H 19$ gene is expressed from the maternal allele. Differential methylation of ICR1 (normally on the paternal allele) mediates differential binding of CTCF and formation of chromatin structures supporting expression of either H19 or IGF2, but not both, under control of distant enhancer sequences (reviewed in Ideraabdullah et $a l^{2}$ ).
CTCF binds to tandem repeat elements in ICR1, and maternally inherited deletion of CTCF-binding elements is associated with hypermethylation of ICR1 and BWS, though in paternal inheritance there is no phenotypic effect. ${ }^{3-6}$ We recently described BWS pedigrees with novel deletions/mutations involving binding sites other than CTCF-binding sites, and in particular binding sites for the OCT4 and SOX2 pluripotency factors, including a point mutation in an OCT4binding site. ${ }^{6}$ In this study, we report another BWS family with a point mutation affecting an OCT-binding DNA sequence.

\section{CASE REPORT}

Case 1 (Figure 1a) was born to non-consanguineous parents following natural conception. In utero he was noted to have bilateral large kidneys and his term birth weight was $5600 \mathrm{~g}$ (>99th centile). He had macroglossia, undescended testes, a minor right-sided ear anomaly with bifid tragus and diastasis recti, and documented episodes of neonatal hypoglycaemia. He had feeding difficulties initially and repeated mild infections. Ultrasound kidney scans revealed unilateral cysts but with no evidence of change into adulthood. His tongue was surgically reduced at 5 years. His development was relatively normal with normal early milestones (sat at 9 months and walked before a year). He was diagnosed with ADHD at school, and although he attended a normal school he finished schooling at the age of 15 years without formal qualifications. Age of puberty was normal. Aged 24

${ }^{1}$ Faculty of Medicine, University of Southampton, Southampton, UK; ${ }^{2}$ Wessex Genetics Service, Southampton University Hospitals Trust, Southampton, UK and Salisbury Hospital NHS Foundation Trust, Salisbury, UK; ${ }^{3}$ Epigenetics in Human Health and Disease Laboratory, Baker IDI Heart and Diabetes Institute, Melbourne, Victoria, Australia; ${ }^{4}$ Northern Genetics Service, Institute of Human Genetics, International Centre for Life, Newcastle upon Tyne, UK

${ }^{*}$ Correspondence: Dr DJG Mackay, Human Genetics and Genomic Medicine group, Faculty of Medicine, Wessex Regional Genetics Laboratory, Salisbury Hospital NHS Foundation Trust, Salisbury SP2 8BJ, UK. Tel: +01 722 425048; Fax: +01 722 331531; E-mail: DJGM@soton.ac.uk

Received 1 March 2011; revised 3 June 2011; accepted 28 June 2011; published online 24 August 2011 
a
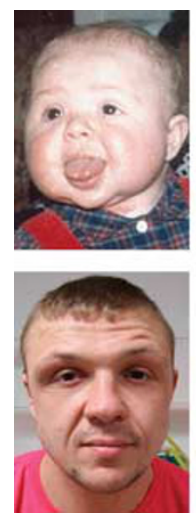

b

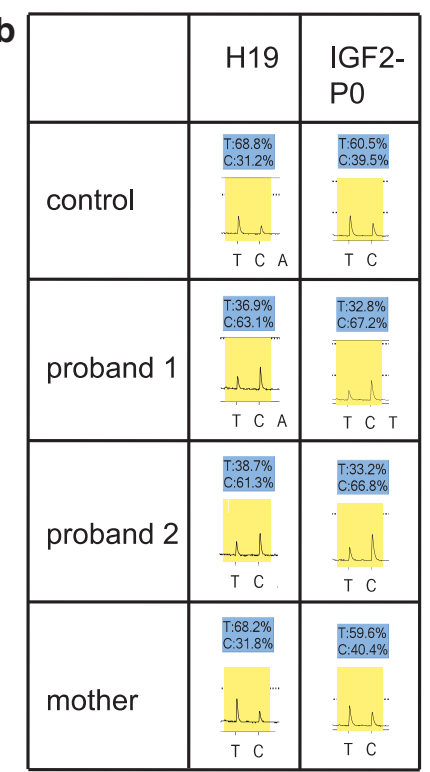

C
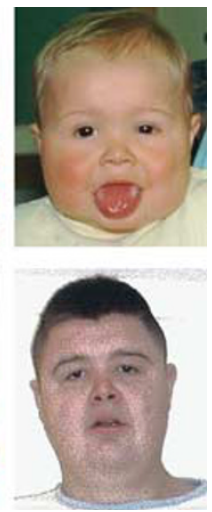

d

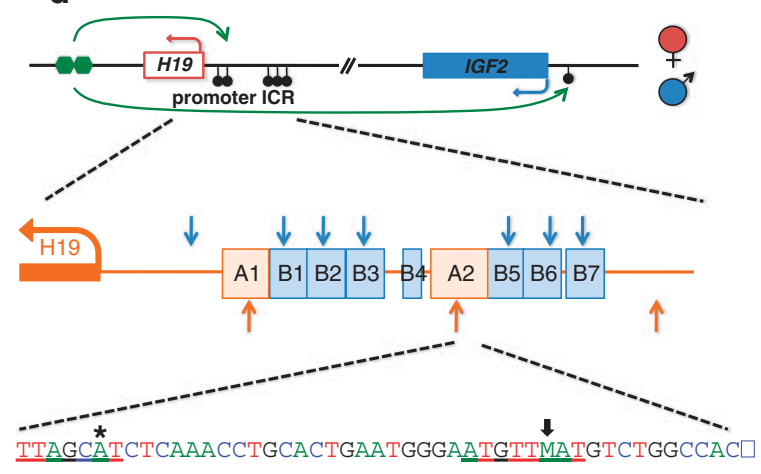

e
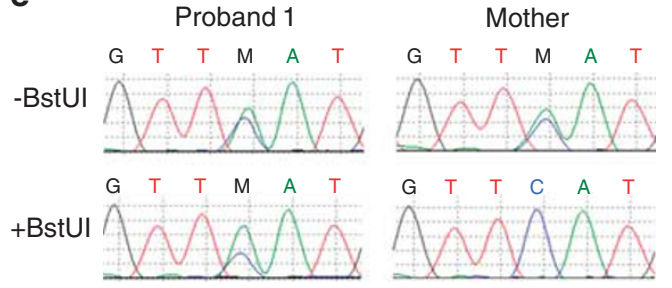

+ BstUI

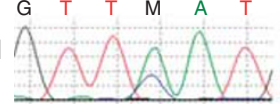

\section{f}

Mouse ES nuclear extract
OCr4/sox2 nuclear extract

0c14/soxi nuclear extract:

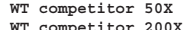

Wut competitor $50 \mathrm{x}$

Mut competitor $200 \mathrm{X}$

WT probe

Mutant probe
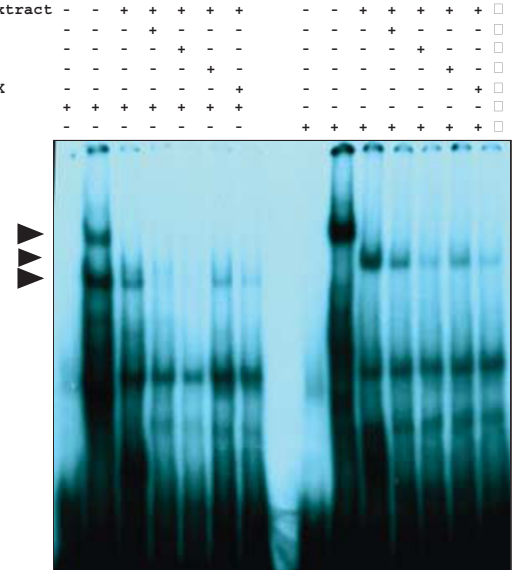

31

23

32

13

32

21

\begin{tabular}{ll}
$\mathrm{D} 11 \mathrm{~S} 4046$ & 21 \\
$\mathrm{D} 11 \mathrm{~S} 1318$ & 31 \\
\hline
\end{tabular}

Figure 1 (a) Upper left: proband 1 aged 3 year; upper right, proband 2 aged 1 year; lower panels, proband 1 aged 24 year. (b) Examples of pyrograms illustrating allele quantification of bisulfite-induced $\mathrm{C} / \mathrm{T}$ polymorphisms within the $\mathrm{H} 19$ and IGF2PO differentially methylated regions. Successive rows illustrate a normal control, proband 1, proband 2 and their mother. The left and right columns illustrate the H19-ICR and IGF2PO, respectively. Each table cell contains a representative pyrogram of the first $\mathrm{C} / \mathrm{T}$ polymorphism quantified by the pyrosequencing reaction. Figures above each pyrogram indicate the proportions of methylated and unmethylated product present in the amplicon. (c) Pedigree of the two probands, and inheritance of microsatellites surrounding H19, illustrating the shared maternal haplotype. (d) Diagram of H19-IGF2-imprinted domain. Top panel: schematic of H19 and ICR1. Differential expression of H19 (maternal allele) and IGF2 (paternal allele) is regulated by ICR1 (the methylated paternal allele is marked with black 'Iollipops'). Differential methylation of the ICR regulates differential access of H19 and IGF2 to shared proximal promoter elements (marked by green hexagons). Second panel: organisation of H19 ICR. The ICR comprises B-type repeats (blue boxes) containing CTCF-binding motifs (blue arrows) interspersed with A-type repeats (orange boxes) and OCT-binding motifs (orange arrows). Third panel: sequence context of mutation chr11:1979624A $>C$ (black arrow); the mutation described by Demars et a/ is marked by an asterisk. (e) Parental origin of mutation. The left and right pairs of electropherograms illustrate proband 1 and his mother; upper and lower pairs illustrate genomic DNA with and without digestion of unmethylated DNA by BstU1. (f) Electrophoretic mobility shift assay of the wild-type and mutant OCT-binding motif. Arrowheads mark protein-DNA complexes.

years, endocrine tests revealed random GH 5 U/l, IGF1 19.4 nM, IGF2 $63.6 \mathrm{nM}, \mathrm{IGF} 1 / \mathrm{IGF} 2$ ratio $3.3(<10)$ and normal glucose tolerance. At 24 years, his height of $204 \mathrm{~cm}$, weight of $119.6 \mathrm{~kg}$ and head circumference of $58.2 \mathrm{~cm}$ were all $\gg 99$ th centile for age. His height was proportionate and his span length $211.5 \mathrm{~cm}$. He had prominent supraorbital ridges and a large mouth, but no ear abnormalities other 
than the bifid right tragus. He had large hands and feet with deepset ingrowing toenails of the halices. Joint movement was not restricted. The rest of the examination was normal with no evidence of asymmetry and a normal neurological examination.

Case 2 (Figure 1a) is the younger brother of case 1. He was born at term following normal conception and an uneventful pregnancy with a birth weight of $6270 \mathrm{~g}$ ( $\gg 99$ th centile). Like his brother he had macroglossia requiring surgical correction, undescended testes, diastasis recti and documented neonatal hypoglycaemia. At 14 months he was found to have mild spasticity of both lower limbs and later required surgical release of a tight left Achilles tendon. By 6 years, mild left sided hemiparesis was apparent, and brain MRI showed a rightsided infarct of perinatal origin. He also has a mild right-sided hemihypertrophy with a leg length discrepancy of $0.5 \mathrm{~cm}$. Routine renal scanning at 14 months identified a right-sided Stage I Wilms' tumour, which was treated with nephrectomy and chemotherapy. $\mathrm{He}$ needed a special educational statement, which was described as having poor concentration and lethargy, and after education at normal school with additional help left without formal qualifications. He has generalised joint hypermobility and significantly restricted mobility owing to a severe planovalgus deformity of the left ankle. Puberty was delayed and now at 22 years he has minimal body hair. Current height is $208 \mathrm{~cm}$ and weight $139.7 \mathrm{~kg}$.

The mother and father of both the boys were born with normal birth weights and final heights of 163 and $183 \mathrm{~cm}$, respectively. Neither had any features of Beckwith-Wiedemann syndrome.

\section{MOLECULAR STUDIES}

Methylation analysis of H19 and IGF2P0 was performed by methylation-specific PCR (MSP) and pyrosequencing as described. ${ }^{7} \mathrm{H} 19$ and IGF2P0 interrogated methylation of cytosines by MSP of the amplicons chr11:1975598-1981477 and chr11:2,126,061-2,126 and 227, while pyrosequencing amplicons spanned chr11:1977650-1977877 and chr11:2,125,904-2,126 and 158, respectively (sequence build NCBI36/hg18); further details are available on request. Methylationspecific MLPA was performed using kit ME-030 v1 (MRC-Holland, Amsterdam, The Netherlands) according to the manufacturer's instructions. Microsatellite analysis of chr1lp15 was performed by standard methods. Array analysis utilised a highly tiled aCGH array as described. ${ }^{8}$ Long-range PCR of $\mathrm{H} 19$ was performed as described. ${ }^{5}$

The H19 ICR and proximal promoter (HG18:chr11:19769781981258) were sequenced by standard methods from a single amplicon of $4281 \mathrm{bp}$; further details are available on request. For some experiments, $200 \mathrm{ng}$ genomic DNA was cleaved using restriction enzyme BstUI (New England Biolabs, Ipswich, MA, USA) following the manufacturer's instructions; then DNA was desalted and concentrated using Amicon $30 \mathrm{~K}$ microconcentrator columns (Millipore, Billerica, MA, USA) before amplification and sequencing.

Electrophoretic mobility shift assay was carried out as described. ${ }^{6}$ Briefly, radiolabelled double-stranded probes were incubated with nuclear extracts from mouse ES cells or from 293FT cells overexpressing human OCT4 and SOX2 proteins, in the presence of wild-type (5'-CTCAAACCTGCACTGAATGGGAATGTTAATGTCTGGCCACTT AAGATTG- $3^{\prime}$ ) or mutated (5'-CTCAAACCTGCACTGAATGGGAAT GTTCA TGTCTGGCCACTTAAGATTG-3') probes as indicated. Reaction mixtures were resolved on non-denaturing $4 \%$ polyacrylamide gel and exposed on film.

\section{MOLECULAR RESULTS}

Molecular testing of blood DNA in probands 1 and 2 indicated ICR 1 hypermethylation: methylation-specific PCR of ICR1 gave methylation indices of 0.23 and 0.27 , respectively, while MS-MLPA gave methylation indices 0.33 and 0.32 , and methylation at the KCNQ1OT1 promoter was normal (data not shown). These results were confirmed by pyrosequencing of ICR1 and IGF2P0 (Figure 1b). Methylation of maternal DNA was normal, and paternal DNA was not available.

The presence of ICR1 hypermethylation in both probands suggested an underlying genetic anomaly. Microsatellite analysis showed that both brothers inherited identical alleles from both parents around ICR1 (Figure 1c), but showed no evidence of allelic imbalance. MS-MLPA revealed no copy number change at $11 \mathrm{p} 15$. Long-range PCR of ICR1 showed no evidence of microdeletions (data not shown). Tiling array-CGH using a highly tiled array spanning the entire imprinted region on $11 \mathrm{p} 15$ revealed no copy number change of any kind (data not shown).

Sanger sequencing of the H19 ICR and proximal promoter revealed a single sequence change: NCBI36:11:g.[1979624A $>C]+[=]$, in both probands and their unaffected mother (Figure 1e). The variant was not found in published databases of sequence variation, nor in 100 normal control samples. To determine the parental origin of the polymorphism, genomic DNA was digested with the methylationsensitive restriction enzyme BstUI, which would be expected to cleave the unmethylated maternal allele of ICR1. As predicted, in both probands both alleles were detectable after restiction digest, as both alleles were methylated. However, in maternal DNA the wild-type allele was undetectable after restriction digest (Figure 1e), indicating that the variant was present on the paternal allele and would be without effect on ICR1.

The sequence change is 29 nucleotides from a mutation recently reported in a BWS pedigree (Family 1, Demars et $a l^{6}$ ). Both variants lie within the A2 repeat of ICR 1 and are predicted to alter binding sites for OCT transcription factors. The functional significance of this variant was tested by the electrophoretic mobility shift assay (EMSA; Figure 1e). With mouse ES cell nuclear extracts the 32P-labelled wild-type probe formed two complexes, whereas only one complex was observed with the 32P-labelled mutated probe. When using cell lysate overexpressing human OCT4 and SOX2 proteins, one complex was identified with both the 32P-labelled wild-type and mutated probes, but the complex obtained with the mutated probe was retarded on the gel. Moreover, the complex obtained with the 32Plabelled wild-type probe was more efficiently competed by an excess of cold wild type than an excess of cold-mutated probe. These data demonstrate that the variant nucleotide alters the binding of nuclear factors, most likely OCT4. Taken together, with its parental origin, this strongly suggests that the variant is the mutation causing BWS in the two probands.

We sequenced DNA from nine other individuals with BWS and H19 hypermethylation, all apparently sporadic, and found no evidence of this mutation or any other novel variant in the H19 ICR or promoter (data not shown).

\section{DISCUSSION}

Although recombinative deletions of B-type repeats in ICR1 have been identified in several BWS pedigrees, ${ }^{3-6}$ and in apparently sporadic Wilms' tumours, ${ }^{9}$ the group of Gicquel were the first to describe a mutation in a pluripotency factor-binding site. ${ }^{6}$ Interestingly, the point mutation in our pedigree, like that described by Demars et $a l^{6}$ disrupted a conserved OCT-binding motif within repeat A2 of ICR1. In a mouse model, A-type motifs binding OCT family proteins were involved in maintaining the unmethylated status of the neighbouring B-type repeats. ${ }^{10}$ Because this function is required only on the maternally inherited allele, neither brother is at the risk of 
transmitting BWS to his offspring, and inheritance by subsequent generations can be monitored molecularly. Therefore, the provision of a concrete molecular diagnosis provides reassurance to the family.

ICR1 hypermethylation accounts for $\sim 10 \%$ of BWS cases. ${ }^{1}$ Approximately $20 \%$ of these have genetic defects, including ICR 1 deletions and OCT or SOX binding site mutations or deletions. In all, $\sim 50 \%$ of patients with maternally transmitted ICR 1 mutations have an apparently sporadic presentation with no family history of BWS. In this study we found no mutations in ICR 1 or the H19 promoter among nine patients with apparently sporadic H19 hypermethylation. More extensive sequencing may identify cis-acting mutations outside the regions already examined. Alternatively, as suggested by the studies of Riccio and co-workers, ${ }^{5}$ ICR1 hypermethylation may arise from a stochastic error in the binding of regulatory proteins during the earliest cell divisions of the embryo. This suggestion receives some support from the observation that even inherited mutations in $\mathrm{H} 19$ do not cause universal somatic hypermethylation, but partial hypermethylation. Either these mutations do not inevitably cause methylation errors in all affected cells, or universal somatic hypermethylation of H19 is incompatible with life, such that the only individuals seen with this disorder are necessarily those with partial rescue from the mutant epigenotype.

The two siblings in this pedigree showed many of the cardinal features of BWS: prenatal overgrowth, macroglossia, neonatal hypoglycaemia, ear dysmorphism, Wilms' tumour and asymmetry. Interestingly, the siblings continued to grow into their twenties, whereas most individuals with BWS show rapid growth in early childhood but decelerating growth in the teens. The height of both probands is $>99$ th centile while adult height in BWS is typically at the upper range of normal. ${ }^{11}$ Identification of similar cases will help determine whether this atypical growth is particularly related to the genotype.

Genetic counselling is increasingly complex for BWS, and this case report illustrates the importance of considering a genetic cause for an epigenetic aberration when giving recurrence risks. In summary, this pedigree confirms that point mutations within ICR1 affecting OCT binding sites cause hypermethylation of the H19 ICR and BeckwithWiedemann syndrome.

\section{CONFLICT OF INTEREST}

The authors declare no conflict of interest.

\section{ACKNOWLEDGEMENTS}

This study was funded by the Newlife Foundation for Disabled Children.

1 Weksberg R, Shuman C, Beckwith JB: Beckwith-Wiedemann syndrome. Eur J Hum Genet 2009; 18: 8-14.

2 Ideraabdullah FY, Vigneau S, Bartolomei MS: Genomic imprinting mechanisms in mammals. Mut Res 2008; 647: 77-85.

3 Sparago A, Cerrato F, Vernucci M, Ferrero GB, Silengo MC, Riccio A: Microdeletions in the human H19 DMR result in loss of IGF2 imprinting and Beckwith-Wiedemann syndrome. Nat Genet 2004; 36: 958-960.

4 Prawitt D, Enklaar T, Gartner-Rupprecht B et al: Microdeletion of target sites for insulator protein CTCF in a chromosome $11 \mathrm{p} 15$ imprinting center in BeckwithWiedemann syndrome and Wilms' tumor. Proc Natl Acad Sci USA 2005; 102: 4085-4090.

5 Cerrato F, Sparago A, Verde G et al: Different mechanisms cause imprinting defects at the IGF2/H19 locus in Beckwith-Wiedemann syndrome and Wilms' tumour. Hum Mol Genet 2008; 17: 1427-1435.

6 Demars J, Shmela ME, Rossignol S et al: Analysis of the IGF2/H19 imprinting control region uncovers new genetic defects, including mutations of OCT-binding sequences, in patients with $11 \mathrm{p} 15$ fetal growth disorders. Hum Mol Genet 2010; 19: 803-814.

7 Mackay DJG, Callaway JLA, Marks SM et al: Hypomethylation of multiple imprinted loci in patients with transient neonatal diabetes is associated with mutations in ZFP57. Nat Genet 2008; 40: 949-951.

8 Poole RL, Baple E, Crolla JA, Temple IK, Mackay DJG: Investigation of a routine cohort referred for molecular cytogenetic analysis using aCGH reveals previously unsuspected anomalies of imprinting. Am J Med Genet 2010; 152A: 1990-1993.

9 Scott RH, Douglas J, Baskcomb L et al: Constitutional $11 \mathrm{p} 15$ abnormalities, including heritable imprinting center mutations, cause nonsyndromic Wilms' tumor. Nat Genet 2008; 40: 1329-1334.

10 Hori N, Nakano H, Takeuchi T et al: A dyad oct-binding sequence functions as a maintenance sequence for the unmethylated state within the H19/lgf2-imprinted control region. J Biol Chem 2002; 277: 27960-27967.

11 Shuman C, Beckwith JB, Smith AC, Weksberg R: Beckwith-Wiedemann syndrome; in Pagon RA, Bird TC, Dolan CR et al, (eds): GeneReviews [Internet]. Seattle: University of Washington, 1993 (http://www.ncbi.nlm.nih.gov/sites/GeneTests/review?db=GeneTests). 